\title{
The challenge of developing universal vaccines
}

\author{
Rino Rappuoli
}

Address: Novartis Vaccines and Diagnostics, Via Fiorentina 1, 53100 Siena, Italy

Email: rino.rappuoli@novartis.com

FI000 Medicine Reports 20II, 3:16 (doi:10.34I0/M3-16)

This is an open-access article distributed under the terms of the Creative Commons Attribution-Non Commercial License (http://creativecommons.org/licenses/by-nc/3.0/legalcode), which permits unrestricted use, distribution, and reproduction in any medium, provided the original work is properly cited. You may not use this work for commercial purposes.

The electronic version of this article is the complete one and can be found at: http://fl000.com/reports/m/3/l6

\begin{abstract}
Antigenic variability of immunodominant antigens is a common mechanism used by pathogens to escape the immune response. Frequently, the proposed solution is a universal vaccine based on conserved antigens present on all strains of the pathogen. Indeed, a lot of progress has been made in the development of vaccines that induce broad immune responses. However, truly universal vaccines are not easy to produce and still face many challenges, mostly because in those pathogens that use antigenic variability to escape the immune response, conserved antigens have been selected by evolution to be poorly immunogenic. This review describes the progress made towards the development of vaccines inducing broad protection against Neisseria meningitidis, influenza, HIV, and Candida and the challenges of developing truly universal vaccines.
\end{abstract}

\section{Introduction}

The most immunogenic molecules on the surfaces of pathogens-the ones that elicit the strongest immune response-are often also the most variable. The influenza virus changes its surface proteins so rapidly that a different vaccine must be manufactured every year. However, the nonimmunogenic part of the surface proteins remains unchanged. To overcome this difficulty, researchers are employing a number of new approaches-from tinkering with the immune system to scouring viral genomes for proteins that are conserved across generations ("reverse vaccinology") - in a quest to create so-called "universal" vaccines. With such vaccines, one jab for each pathogen will protect us from all current and future variations of each disease, and maybe even from different families of related pathogens. But why has it been difficult to create such vaccines, and what progress are we making?

\section{Serogroup B meningococcus}

Neisseria meningitidis is a Gram-negative bacterium that has been divided into 12 serogroups classified according to the chemical composition of the polysaccharide capsule, five of which cause disease in humans: A, B, C, W135, and Y. Antibodies to the capsular polysaccharides protect from disease, but the capsular polysaccharides are not immunogenic, especially in children, because they are T-cell-independent antigens. The poor immunogenicity of the A, C, W135, and Y polysaccharides was overcome by conjugating the polysaccharides to a protein able to engage the T cells [1] (Figure 1). Using this technology, vaccines against serogroups A, C, W135, and Y were developed and licensed.

However, attempts to make a vaccine for serogroup B meningococcus, which causes approximately $50 \%$ of the global cases of meningococcal meningitis outside Africa (where serogroup A predominates), failed many times for two reasons. The first is that the capsular polysaccharide, which is the most conserved antigen, is nonimmunogenic even when conjugated to a protein carrier because it has a chemical composition identical to a self antigen-the polysialic acid present in human glycoproteins (Figure 2).

The second reason is that PorA, the most abundant and most immunogenic protein in the envelope of the bacterium, is highly variable in sequence and induces a strong protective immune response against the homologous strain only. Analysis of the feasibility of a universal vaccine made using this approach in the United States 
Figure I. Schematic representation of Neisseria meningitidis serotypes $A, C, W, Y$

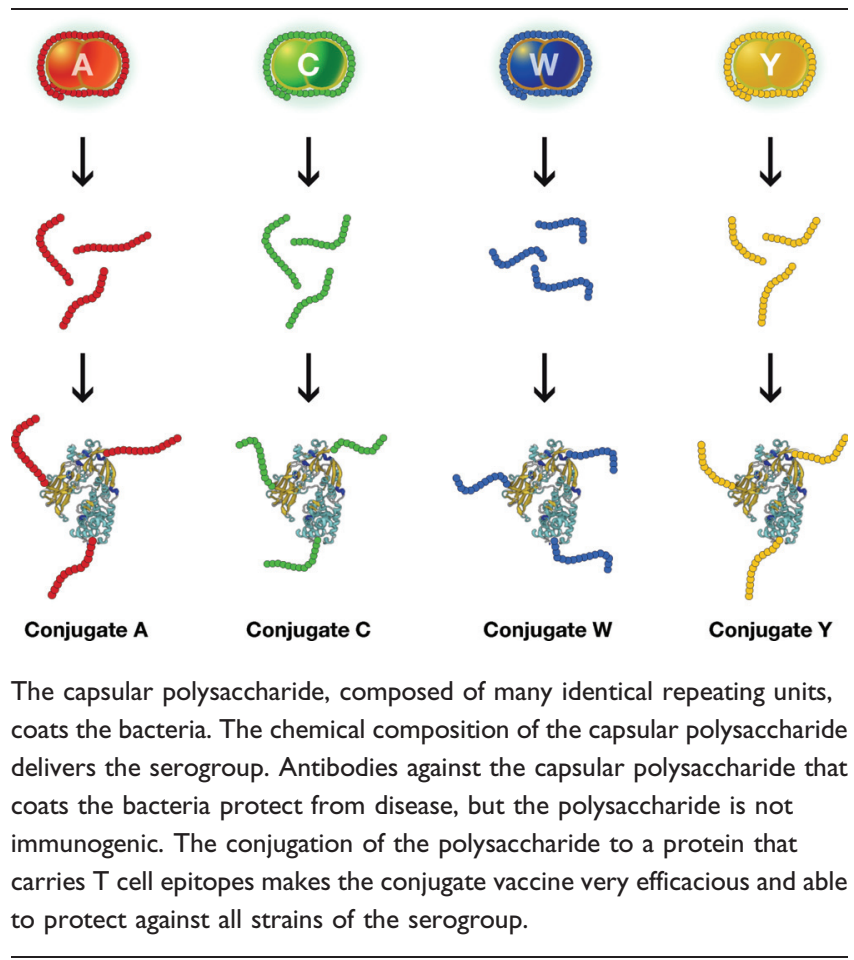

Figure 2. Schematic representation of Neisseria meningitidis serogroup B (MenB)

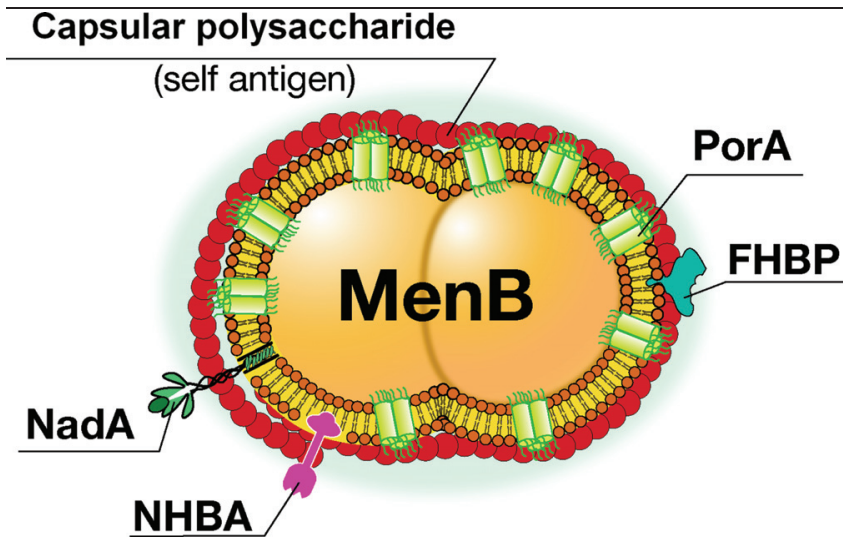

The capsular polysaccharide is a self antigen that cannot be used to make a vaccine. The most abundant antigen is PorA, which is variable and induces only strain-specific protection. A vaccine inducing broad protection was developed using reverse vaccinology to mine the genome and identify less abundant but more conserved antigens such as FHBP (factor H-binding protein), NadA (Neisseria adhesin A), and NHBA (Neisseria heparin-binding antigen).

revealed that a vaccine made from one strain would cover a maximum of $27 \%$ of the strains in the United States and that at least 20 different vaccine strains were necessary to make a multicomponent vaccine covering $80 \%$ of the bacterial population. This complex vaccine was found to be beyond technical feasibility [2].

The solution for a broadly cross-reactive vaccine was found by searching the genome of the bacterium for antigens that being neither too abundant nor immunodominant would not be subjected to strong selective pressure, and would therefore be more conserved. The search was successful, and out of 2,158 genes, 28 were found to code for protective antigens with these properties [3]. Finally, three of them were used in a vaccine formulation that also contained outer membrane vesicles from a strain producing the PorA P.1.4. The vaccine has now finished Phase III clinical trials and is being submitted for regulatory approval to the European Medicinal Agency.

The three antigens identified by the genome search were: Neisseria heparin-binding antigen (NHBA), which is present in all strains and induces a bactericidal response against all strains that produce enough quantity of the protein; the factor H-binding protein (FHBP), which is present in most of the strains, but due to sequence diversity has been divided into three variants that do not induce cross-neutralization (variant 1 , which is present in more than $60 \%$ of all strains, was included in the vaccine); and Neisseria meningitidis adhesin A (NadA), which is present in approximately $50 \%$ of the strains but, when present, is a good target for bactericidal antibodies [4] (Figure 2). A recent publication has analyzed the predicted coverage of the global diversity of meningococcus $\mathrm{B}$ strains of this vaccine. To do so, the amount of each antigen and its antigenic distance from the vaccine antigen were measured using a novel enzyme-linked immunosorbent assay (ELISA) named "Meningococcal Antigen Typing System" (MATS) [5]. The analysis revealed that adults and infants immunized with the vaccine would be protected from disease caused by $86 \%$ and $77 \%$ of the global strains, respectively. Interestingly, more than $50 \%$ of the strains were independently covered by two or more of the vaccine antigens, suggesting that vaccine escape mutants should be unlikely. In conclusion, in the case of serogroup B meningococcus, the combination of several antigens discovered by reverse vaccinology has allowed the development of a vaccine that is predicted to cover most of the strains of this highly variable bacterium.

\section{Influenza}

Currently, more than 100 World Health Organization (WHO) centers worldwide monitor the circulation of influenza strains and, by using this information every year, the experts make a prediction of which strains are likely to circulate during the following year. Based on this prediction, new vaccine strains are developed and provided in 
February and September to vaccine manufacturers. So currently we use a new vaccine strain for each variant of the seasonal influenza virus. This also applies for pandemic strains. For example, vaccines were made against several variables of the pandemic influenza strain $\mathrm{H} 5 \mathrm{~N} 1$ clade $\mathrm{O}$ isolated in Hong Kong in 1997, clade I isolated in Vietnam in 2004, and clade II isolated in Indonesia in 2005. In developing a pandemic vaccine, it is advantageous to use the latest virus isolate if possible, but this can cause delays. For example, during the $2009 \mathrm{H} 1 \mathrm{~N} 1$ pandemic, the production and characterization of the vaccine strain using the pandemic virus delayed the manufacturing of the annual vaccine by a couple of months so that it only became available in large quantities after the peak of the circulating virus. Clearly, a universal vaccine able to immunize against any form of influenza would be very welcome. Is this possible? Figure 3 summarizes what we know about influenza today.

Current practice has been established based on past scientific knowledge: each influenza strain variant needs a different vaccine. In the last few years we have learned that using oil-in-water adjuvants such as MF59 increases the titer of the antibody responses, the number of memory $\mathrm{T}$ and memory B cells, and broadens the B cell epitopes recognized by the antibodies so that the vaccine is able to cover not only the immunizing strain, but also the strains that are closely related $[6,7]$. Following these findings, it is now possible that we could use one vaccine to cover all $\mathrm{H} 5$ strains, one vaccine to cover all swine $\mathrm{H} 1 \mathrm{~N} 1$ strains, and one vaccine to cover related human H1N1 strains. Therefore, we could already use the same vaccines for

Figure 3. The path towards broadly protective influenza vaccines

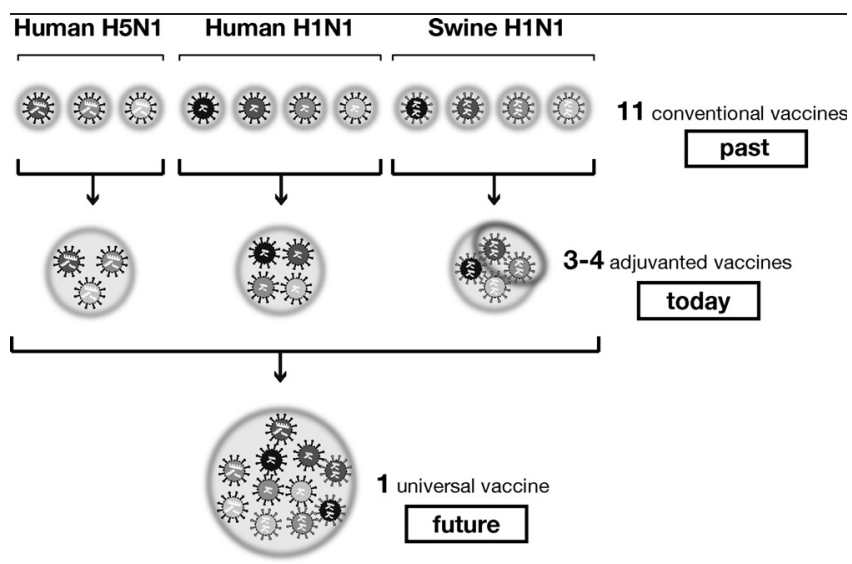

Up to this point, one vaccine has been used for each influenza strain so that II different vaccines are needed to cover the II strains shown in the figure. Today, using oil-in-water adjuvants such as MF59, it is possible to vaccinate against the II strains using only 3 or 4 vaccines. The dream for the future is to develop a universal vaccine able to protect from all strains. closely related strains and change the vaccine only when a major antigenic change occurs. This approach would not be the much-dreamed-of "universal" vaccine, but it would certainly be a major step forward compared with the present situation.

The dream is to create a truly universal vaccine to be used against all strains of influenza, but is this going to become a reality, and if so, when? At this point it is difficult to make predictions, and although tremendous progress has been made in this direction, we still do not even know whether this is actually possible. Many of the proposed solutions for a universal influenza vaccine suggest using conserved antigens such as the $\mathrm{M} 1$ and $\mathrm{M} 2$ proteins. While using these proteins makes sense scientifically, it is worth considering that the $\mathrm{M} 1$ protein is already present in most vaccines and little progress has been made using M2 since it was originally proposed as a vaccine antigen more than 10 years ago [8]. Recently, universal neutralizing antibodies have been isolated against the conserved HA2 region of hemagglutinin (HA), raising the expectation that a universal vaccine may be developed using this region [9] (Figure 4).

Figure 4. Schematic representation of the influenza hemagglutinin trimer showing the conserved $\mathrm{HA} 2$ region and the variable $\mathrm{HAl}$ regions

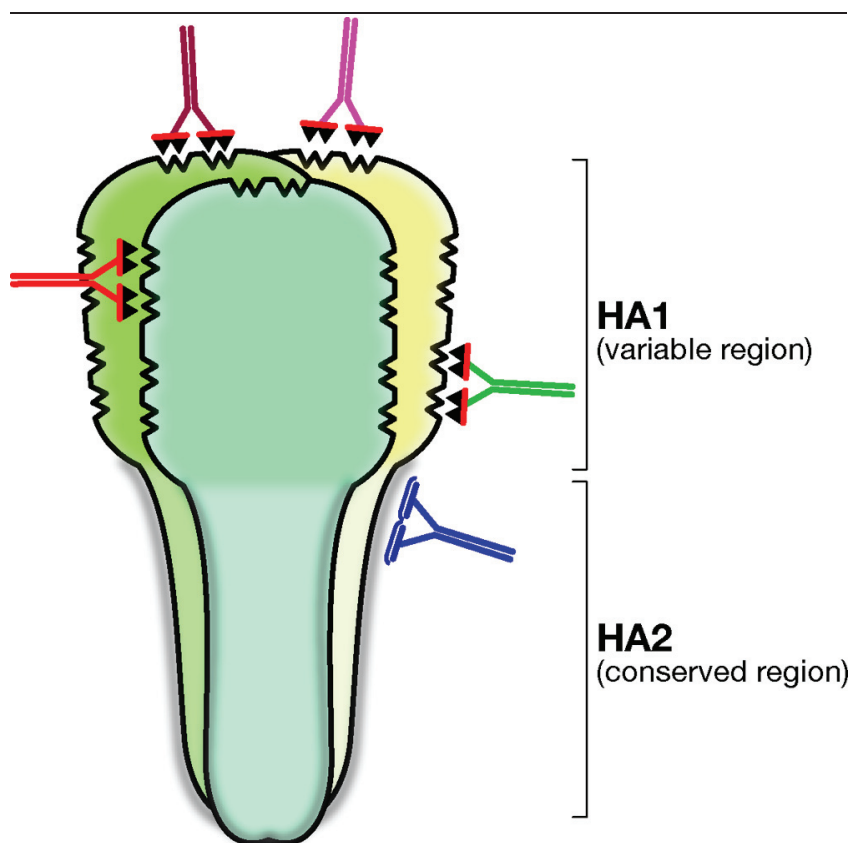

The figure shows that the contact surface of the poorly immunogenic HA2 region is flat and may be difficult to bind with high affinity. On the other hand, the immunodominant HAI region has many cavities that allow antibody binding with high affinity. 
However, universal neutralizing antibodies are rare, have low affinity, and cannot be induced in large quantities during infection or vaccination. The most advanced work in this direction showed that priming with plasmid DNA encoding H1N1 influenza hemagglutinin and boosting with seasonal vaccine or replication-defective adenovirus 5 vector encoding hemagglutinin can stimulate some broadly neutralizing antibodies against this region [10]. However, even in this case, these antibodies represent a small fraction of the total neutralizing antibodies and had to be preabsorbed with a protein without the stem of hemagglutinin in order to be analyzed. The question is why are the most conserved universal epitopes so poorly immunogenic? Clearly, these epitopes have been strongly selected during evolution to be nonimmunogenic. In fact, variant strains of influenza would not cause disease in previously exposed individuals if they were able to induce a strong protective response. The molecular reasons behind the poor immunogenicity are less clear. Maybe they evolved to have epitopes that coincide with a hole in a B cell germline repertoire, maybe the epitope is hard to reach [11], or maybe there is an even simpler reason. For instance, looking at Figure 4 it is clear that the surface of the HA2 region is simply flat and that it is very difficult for the antibodies to find something to grab and bind to with high affinity. This is in marked contrast with the antibodies that bind the globular head of hemagglutinin, which is the most immunogenic but variable region in hemagglutinin. This region is full of cavities and this makes it easy for the antibodies to grab and stick with high affinity. Therefore, to make a universal vaccine against the HA2 region of the influenza hemagglutinin, we may need to "teach" the antibodies to bind and climb a flat slippery surface, and this may not be easy.

\section{HIV}

In the case of HIV, in order to make a vaccine able to protect against all the HIV variants, scientists focused their attention on the CD4 binding site, which is the most conserved part of the HIV envelope protein gp120. The virus cannot change this region without committing suicide and therefore it would be an ideal epitope for a universal vaccine. Indeed, two beautiful papers recently published in Science showed that rare broadly neutralizing antibodies against this region can be found in infected patients and showed the structural basis for the broad neutralization $[12,13]$. A similar paper also published in Nature some years ago showed the structural details of another broadly neutralizing antibody, B12, against this region [14]. These publications, which represent the most advanced state of the art in the field, have made incredible progress in the understanding of the basic mechanisms of broad HIV neutralization, but unfortunately have not yet been able to instruct us on how to design an immunogen able to induce broadly neutralizing antibodies to HIV. As in the case of influenza and meningococcus $\mathrm{B}$, the most conserved epitope has been selected during evolution to be poorly immunogenic, and therefore antibodies against it are very rare, but why? A quick look at the crystal structure of the B12 antibody gp120 complex shows that the antibody binds the epitope only with the heavy chain, because the epitope is recessed and not easily accessible (Figure 5). So, while influenza makes the HA2 epitope nonimmunogenic by making it a flat surface, HIV made the $\mathrm{CD} 4$ binding site nonimmunogenic by hiding this region in a remote and hardly accessible site.

\section{Candida and other pathogenic fungi}

Opportunistic pathogens such as Candida, Aspergillus, and Cryptococcus are not dangerous for healthy individuals, but under particular conditions where the immune system is weakened they can become fierce pathogens and cause high mortality. Given the increasing importance of opportunistic pathogens today, vaccines against these fungi would be desirable. Fungal vaccine development has mostly used cell wall extracts, which contain polysaccharides that are

Figure 5. Schematic representation of the HIV CD4 binding site that is conserved in all HIV envelopes, but is recessed and poorly immunogenic

Conserved CD4 epitope

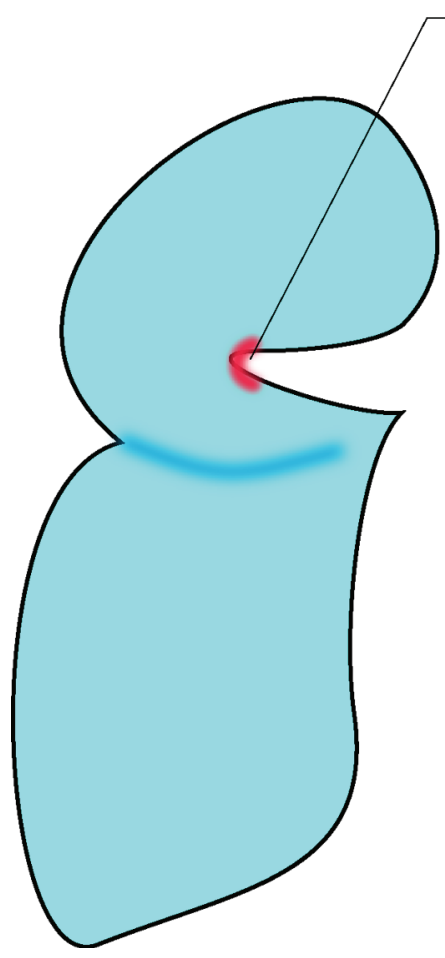


generally poorly immunogenic, and protection in animal models has been controversial. In fact, antibody-mediated protection has been difficult to demonstrate because inhibitory and protective antibodies seem to be equally induced. However, it was encouraging to see recently that the monoclonal antibody $2 \mathrm{G} 8$ was isolated and able to control infections caused by two fungi, Candida and Cryptococcus. The antibody was shown to recognize beta 1,3 glucan, a component of the cell wall that is present in all fungi, and to be able to inhibit the growth of the Candida hyphae in vitro [15]. Given the poor immunogenicity of the beta glycan, it was decided to conjugate laminarin (a product derived from algae that contains mostly beta glycan) to the carrier protein CRM197. This proved to be another success-the CRM197-laminarin conjugate vaccine was immunogenic and protected against infection with Candida and Aspergillus [16].

It is therefore possible that the conjugation technology that has been so successful in making immunogenic bacterial polysaccharides may be useful in the development of universal "cross-kingdom" vaccines able to protect against multiple fungal infections.

\section{Conclusions and future outlook}

Highly conserved antigens of successful pathogens are usually poorly immunogenic and have been selected during evolution to avoid engaging the immune system. In some cases, new technologies have been able to overcome the poor immunogenicity. A successful example is bacterial capsular polysaccharides. In this case, the conjugation technology allowed the development of "universal vaccines" able to cover all strains belonging to one serogroup. A similar conjugation technology may be used to develop a vaccine against Candida and all pathogenic fungi. A genomic approach such as reverse vaccinology was necessary to find good antigens suitable for the development of a vaccine against meningococcus $\mathrm{B}$. In the case of influenza, quantum jumps were made by using innovative adjuvants. However, many challenges are still ahead of us. Making an HIV vaccine, a truly universal vaccine against influenza, a vaccine against malaria, and a vaccine against rhinoviruses, for example, is still far away. The main approach used in these cases has been the use of structure-based antigen design to render the conserved epitopes immunogenic. After several challenging years, we have made a lot of progress in the scientific understanding of conserved regions, especially in the case of HIV and influenza; however, little progress has been made in engineering immunodominance in these regions. Therefore it is important that we also look for alternative technical solutions. An alternative approach could also be to use structure-based antigen design to
Figure 6. Structure-based engineering of immunodominant epitopes

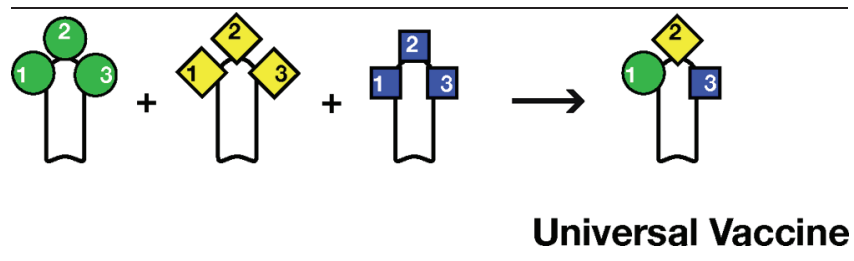

Structure-based engineering of immunodominant epitopes can be used to design protective epitopes from different variants in one molecule and develop new immunogens able to induce broad protection against a species of the pathogen. In the figure, one antigen is engineered to center protective epitopes from different antigenic variants and induce universal immunity.

engineer immunodominant epitopes to cover multiple strains as described in Figure 6. Preliminary data suggest that such an approach may be feasible at least in some cases.

\section{Abbreviation \\ HA, hemagglutinin.}

\section{Competing interests}

The author declares that he has no competing interests.

\section{Acknowledgments}

The author is grateful to Giorgio Corsi for the artwork and to Catherine Mallia for editorial assistance.

\section{References}

I. Costantino P, Viti S, Podda A, Velmonte MA, Nencioni L, Rappuoli R: Development and phase I clinical testing of a conjugate vaccine against meningococcus $A$ and $C$. Vaccine 1992, 10:69|-8.

2. Tondella ML, Popovic T, Rosenstein NE, Lake DB, Carlone GM, Mayer LW, Perkins BA: Distribution of Neisseria meningitidis serogroup $B$ serosubtypes and serotypes circulating in the United States. The Active Bacterial Core Surveillance Team. J Clin Microbiol 2000, 38:3323-8.

3. Pizza $M$, Scarlato $V$, Masignani $V$, Giuliani $M M$, Aricò $B$, Comanducci M, Jennings GT, Baldi L, Bartolini E, Capecchi B, Galeotti CL, Luzzi E, Manetti R, Marchetti E, Mora M, Nuti S, Ratti G, Santini L, Savino S, Scarselli M, Storni E, Zuo P, Broeker M, Hundt E, Knapp B, Blair E, Mason T, Tettelin H, Hood DW, Jeffries AC, et al.: Identification of vaccine candidates against serogroup $B$ meningococcus by whole-genome sequencing. Science 2000, 287: $1816-20$.

4. Giuliani MM, Adu-Bobie J, Comanducci M, Aricò B, Savino S, Santini L, Brunelli B, Bambini S, Biolchi A, Capecchi B, Cartocci E, Ciucchi L, Di Marcello F, Ferlicca F, Galli B, Luzzi E, Masignani V, Serruto D, Veggi D, Contorni M, Morandi M, Bartalesi A, Cinotti V, Mannucci D, Titta F, Ovidi E, Welsch JA, Granoff D, Rappuoli R, Pizza M: A universal vaccine for serogroup $B$ meningococcus. Proc Natl Acad Sci U S A 2006, 103:10834-9.

5. Donnelly J, Medini D, Boccadifuoco G, Biolchi A, Ward J, Frasch C, Moxon ER, Stella M, Comanducci M, Bambini S, Muzzi A, Andrews W, Chen J, Santos G, Santini L, Boucher P, Serruto D, Pizza M, Rappuoli R, Giuliani MM: Qualitative and quantitative assessment of meningococcal antigens to evaluate the potential strain coverage of protein-based vaccines. Proc Natl Acad Sci U S A 2010, 107:19490-5. 
6. Galli G, Medini D, Borgogni E, Zedda L, Bardelli M, Malzone C, Nuti S, Tavarini S, Sammicheli C, Hilbert AK, Brauer V, Banzhoff A, Rappuoli R, Del Giudice G, Castellino F: Adjuvanted H5N I vaccine induces early CD4+ $T$ cell response that predicts long-term persistence of protective antibody levels. Proc Natl Acad Sci U S A 2009, 106:3877-82.

7. Khurana S, Chearwae W, Castellino F, Manischewitz J, King LR, Honorkiewicz A, Rock MT, Edwards KM, Del Giudice G, Rappuoli R, Golding H: Vaccines with MF59 adjuvant expand the antibody repertoire to target protective sites of pandemic avian $\mathrm{H} 5 \mathrm{~N}$ I influenza virus. Sci Transl Med 2010, 2:15ra5.

8. Neirynck S, Deroo T, Saelens X, Vanlandschoot P, Jou WM, Fiers W: A universal influenza $A$ vaccine based on the extracellular domain of the M2 protein. Nat Med 1999, 10:1 I 19-20.

9. Ekiert $D C$, Bhabha $G$, Elsliger $M A$, Friesen $R H$, Jongeneelen $M$, Throsby M, Goudsmit J, Wilson IA: Antibody recognition of a highly conserved influenza virus epitope. Science 2009, 324:246-5I.

FI000 Factor 10

Evaluated by Patrick C. Wilson 2 I Jul 20I I, Rino Rappuoli 26 Jul 201 I

10. Wei CJ, Boyington JC, McTamney PM, Kong WP, Pearce MB, Xu L, Andersen H, Rao S, Tumpey TM, Yang ZY, Nabel G]: Induction of broadly neutralizing HINI influenza antibodies by vaccination. Science 2010, 329:1060-4.

FI000 Factor 10

Evaluated by Deepta Bhattacharya 19 Aug 2010, Patrick C. Wilson 21 Jul 20II, Rino Rappuoli 26 Jul 201 I

II. Kwong PD, Wilson IA: HIV-I and influenza antibodies: seeing antigens in new ways. Nat Immunol 2009, 10:573-8.

FI000 Factor 6

Evaluated by Rino Rappuoli 26 Jul 2011

12. Wu X, Yang ZY, Li Y, Hogerkorp CM, Schief WR, Seaman MS, Zhou T, Schmidt SD, Wu L, Xu L, Longo NS, McKee K, O'Dell S, Louder MK, Wycuff DL, Feng Y, Nason M, Doria-Rose N, Connors M, Kwong PD, Roederer M, Wyatt RT, Nabel GJ, Mascola JR: Rational design of envelope identifies broadly neutralizing human monoclonal antibodies to HIV-I. Science 20I0, 329:856-6I.

FI000 Factor 17

Evaluated by Hanneke Schuitemaker 19 Jul 2010, Sheena McCormack 03 Aug 2010, Eric Hunter 08 Sep 2010, Leo Stamatatos 29 Sep 2010

13. Zhou T, Georgiev I, Wu X, Yang ZY, Dai K, Finzi A, Kwon YD, Scheid JF, Shi W, Xu L, Yang Y, Zhu J, Nussenzweig MC, Sodroski J, Shapiro L, Nabel GJ, Mascola JR, Kwong PD: Structural basis for broad and potent neutralization of HIV-I by antibody VRCOI. Science 2010, 329:8II-7.

FI000 Factor 8

Evaluated by Rino Rappuoli 26 Jul 2011

14. Zhou T, Xu L, Dey B, Hessell AJ, Van Ryk D, Xiang SH, Yang X Zhang MY, Zwick MB, Arthos J, Burton DR, Dimitrov DS, Sodroski J, Wyatt R, Nabel G], Kwong PD: Structural definition of a conserved neutralization epitope on HIV-I gpI20. Nature 2007, 445:732-7.

FI000 Factor II

Evaluated by Guido Silvestri 27 Mar 2007, James Tartaglia 29 Mar 2007

15. Torosantucci A, Chiani P, Bromuro C, De Bernardis F, Palma AS, Liu Y, Mignogna G, Maras B, Colone M, Stringaro A, Zamboni S, Feizi T, Cassone A: Protection by anti-beta-glucan antibodies is associated with restricted beta-I,3 glucan binding specificity and inhibition of fungal growth and adherence. PLoS One 2009, 4:e5392.

16. Pietrella D, Rachini A, Torosantucci A, Chiani P, Brown AJ, Bistoni F, Costantino P, Mosci P, d'Enfert C, Rappuoli R, Cassone A, Vecchiarelli $A$ : $A$ beta-glucan-conjugate vaccine and anti-betaglucan antibodies are effective against murine vaginal candidiasis as assessed by a novel in vivo imaging technique. Vaccine 2010, 28:1717-25.

FI000 Factor 6

Evaluated by Rino Rappuoli 26 Jul 2011 